\title{
'Jinhuang' Yellow-leaf Crape Myrtle
}

Shu'an Wang

Southern Modern Forestry Collaborative Innovation Center/College of Biology and the Environment, Nanjing Forestry University, Nanjing 210037, China; and Institute of Botany, Jiangsu Province and Chinese Academy of Sciences, Nanjing 210014, China

\author{
Rutong Yang, Peng Wang, Qing Wang, Linfang $\mathbf{L i}$, and $\mathbf{Y a ~ L i ~}^{1}$ \\ Institute of Botany, Jiangsu Province and Chinese Academy of Sciences, \\ Nanjing 210014, China
}

\section{Zengfang Yin ${ }^{1}$}

Southern Modern Forestry Collaborative Innovation Center/College of Biology and the Environment, Nanjing Forestry University, Nanjing 210037, China

Additional index words. Lagerstroemia indica, ornamental breeding, mutant, semidwarf shrub

Crape myrtle (Lagerstroemia indica), a member of the family Lythraceae, originated in China and historical records of its cultivation in the country date back to 1700 years ago, well before its cultivation in other areas in the world (Zhang, 1991). Known for its longlasting flowers in midsummer, wide range of flower colors (white, red, purple, pink, and their combined variants), extensive diversity of growth habits (miniature shrub to large tree), and appealing barks (cinnamon colored to gray colored), $L$. indica is a key parent for crape myrtle breeding (Cabrera, 2002). Many crape myrtle cultivars are now widely used in Chinese landscaping (Min et al., 2008). In the past 50 years, most breeding efforts have focused on resistance to diseases and in combinations with other desirable horticultural traits (Pooler, 2006; Pooler and Dix, 1999). Many cultivars are available in a broad range of growth habits to service various landscape objectives (Egolf, 1986, 1990; Knox, 2000). In recent years, five dark leaf crape myrtle cultivars (e.g., Ebony Embers, Ebony Fire, Ebony Flame, Ebony Glow, and Ebony and Ivory) have been bred (Pounders et al., 2013). Although a large number of new crape myrtle cultivars have been released through breeding and selection efforts since the 1960s, no yellow leaf crape myrtle cultivars are available. Here, we report a new yellow leaf crape myrtle cultivar named as Jinhuang.

\footnotetext{
Received for publication 23 Feb. 2016. Accepted for publication 9 Mar. 2016.

This work was supported by Agricultural science and technology achievements transformation of the Ministry of science and technology funds (2013GB2C100185), Agricultural science and technology independent innovation fund of Jiangsu province (CX(14)2032), Sanxin forestry engineering of Jiangsu province (LYSX [2015]13), and the doctorate fellowship foundation of Nanjing Forestry University.

Shu'an Wang and Rutong Yang have contributed equally to this work.

${ }^{1}$ Corresponding author. E-mail: yalicnbg@163. com or zfyin@njfu.edu.cn.
}

as smaller shrubs. The cultivar is propagated by hardwood stem cuttings treated with $800 \mathrm{ppm}$ indole butyric acid under intermittent misting systems in the early spring. 'Jinhuang' can be grafted successfully on L. indica or Lagerstroemia fauriei in the early spring.

\section{Comparative Data}

Performance in the field. 'Jinhuang' and the control cultivar Fenjing were grown in the nursery of CNBG in 2014. The experiment was arranged into a completely randomized design with three replications and five cutting grown plants per replication. Phenotypic traits of plants were measured in July. The Dunnett's test of data was analyzed by IBM SPSS Statistics 19.

Leaf color of 'Jinhuang' was yellow (25A) in spring, and yellow (1A) in summer (Fig. 3). Leaf color of the control cultivar Fenjing was green (140C) in spring, and dark green (131A) in summer (Fig. 3). Branche number of 'Jinhuang' was significantly more than 'Fenjing' (Table 1). However, plant height and ground diameter of 'Jinhuang' were decidedly less than 'Fenjing' (Table 1). Petal length, petal width, and claw length of 'Jinhuang' were slightly less than 'Fenjing' (Table 1).

\section{Availability}

hardwood cuttings. A total of 12 plants of the mutant were continuously evaluated in the nursery of CNBG during 2004 through 2012. We found that leaf color of all clones was stable over the past 8 years. The cultivar Jinhuang was authorized by the state forestry administration of the People's Republic of China in 2014.

\section{Description}

Lagerstroemia indica 'Jinhuang' is a deciduous, semidwarf shrub that has grown $1.7 \mathrm{~m}$ high and $1.8 \mathrm{~m}$ wide at the 11 th year (Fig. 1). Leaf color is yellow (25A, Royal Hort. Soc., 2001) in spring, yellow yellow (1A) in summer. Leaves are 3.3 to $5.5 \mathrm{~cm}$ long, and 2.2 to $3.7 \mathrm{~cm}$ wide, with a 2- to 4-mm-long petiole. The young shoots are red-purple (60A). The young branches are grey-brown (166D) and turn to light grey (166C) when they are older. Plants bloom during mid-July through September. Flower panicles are 9 to $18 \mathrm{~cm}$ long, 7.5 to $17 \mathrm{~cm}$ wide, with 15 to 60 bright pink (N57C), 3.8-to 4.5-cm-diameter flowers (Fig. 2). Petals are deep pink (N57B), 12 to $15 \mathrm{~mm}$ long, 8 to $10 \mathrm{~mm}$ wide, and clawed with slight crinkled. 'Jinhuang' is suitable for gardens, landscapes in restricted spaces, and container plants for patios or paved public areas due to its dwarf plant habit and leaf color.

\section{Culture}

'Jinhuang' is adapted to accepted cultural practices for $L$. indica and its cultivars, which thrive in diverse soil and climatic conditions. They grow and flower best in half sun with adequate moisture and balanced fertility. Plants are amenable to pruning and can be maintained

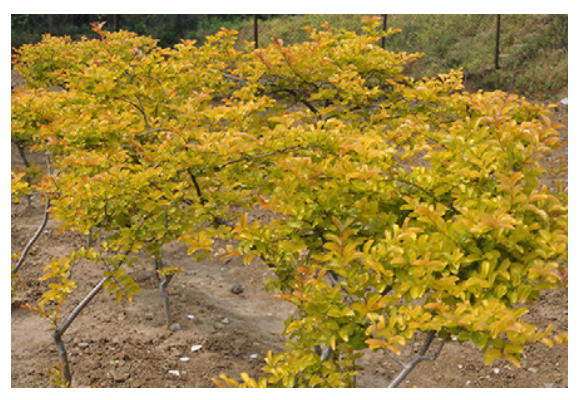

Fig. 1. Eight-year-old cutting grown plants of 'Jinhuang' crape myrtle.

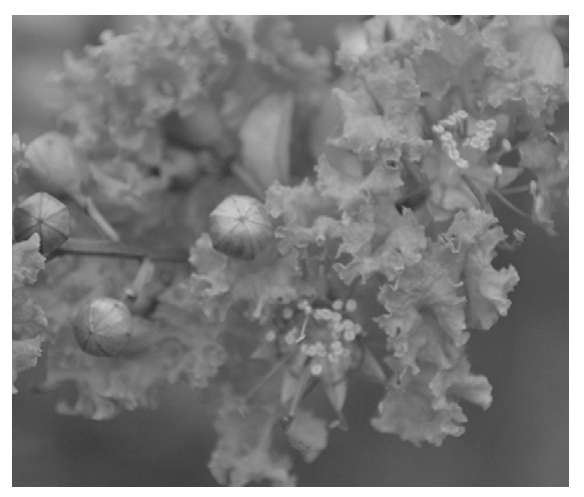

Fig. 2. Flowers of 'Jinhuang' crape myrtle. 




Fig. 3. (A) Spring and (B) summer leaf color of 'Jinhuang' (L) and 'Fenjing' (R) crape myrtles.

Table 1. Phenotypic trait of 'Jinhuang' and 'Fenjing'.

\begin{tabular}{lrc}
\hline Trait & \multicolumn{1}{c}{ 'Fenjing' } & \multicolumn{1}{c}{ 'Jinhuang' } \\
\hline Plant height & $72.8767 \pm 3.01$ & $30.67 \pm 4.76^{* *}$ \\
Branche number & $11.33 \pm 2.94$ & $17.67 \pm 2.73^{* *}$ \\
Ground diameter & $1.05 \pm 0.11$ & $0.60 \pm 0.07^{* *}$ \\
Crown diameter & $4.22 \pm 0.16$ & $4.14 \pm 0.11$ \\
Petal length & $1.80 \pm 0.13$ & $1.79 \pm 0.02$ \\
Petal width & $1.81 \pm 0.02$ & $1.80 \pm 0.01$ \\
Claw length & $0.89 \pm 0.01$ & $0.88 \pm 0.01$ \\
\hline
\end{tabular}

**Significance level was $P<0.01$ by Dunnett's test.

${ }^{z}$ Measured stem at $5 \mathrm{~cm}$ above the ground.

\section{Literature Cited}

Cabrera, R.I. 2002. Evaluating and promoting the cosmopolitan and multipurpose Lagerstroemia. XXVI International Horticultural Congress, Toronto, Canada, 177-184.

Egolf, D.R. 1986. 'Acoma', 'Hopi', 'Pecos', and 'Zuni' Lagerstroemia. HortScience 21:1250-1252.

Egolf, D.R. 1990. 'Caddo' and 'Tonto' Lagerstroemia. HortScience 25:585-587.

Knox, G. 2000. Crape myrtles in Florida. UF Ext. Bul. ENH 52.

Min, W., S. Ping, X.X. Ren, and Q.X. Zhang. 2008. Recent advances in Lagerstroemia indica resources and breeding. Shandong For. Sci. Technol. 175:66-68. (In Chinese).

Pooler, M.R. 2006. 'Arapaho' and 'Cheyenne' Lagersiroemia. HortScience (in press).

Pooler, M.R. and R.L. Dix. 1999. 'Chickasaw', 'Kiowa', and 'Pocomoke' Lagerslroemia. HortScience 34:361-363.

Pounders, C., B.E. Scheffler, and T.A. Rinehart. 2013. 'Ebony Embers', 'Ebony Fire', 'Ebony Flame', 'EbonyGlow', and 'Ebony and Ivory' Darkleaf crape myrtles. HortScience 48:1568-1570.

Royal Horticultural Society. 2001. RHS colour chart. 4th ed. Royal Hort. Soc., London, UK.

Zhang, Q.X. 1991. Studies on cultivars of crape myrtle (Lagerstroemia indica) and their uses in urban greening. Beijing For. Univ. 18:57-66. (In Chinese). 